UCRL-ID-118629

\title{
Conjugate Gradient MATHEW
}

\author{
Gayle Sugiyama \\ Robert L. Lee \\ Hoyt Walker \\ Regional Atmospheric Sciences Division \\ Lawrence Livermore National Laboratory \\ Livermore, California 94551 USA
}

September 1994

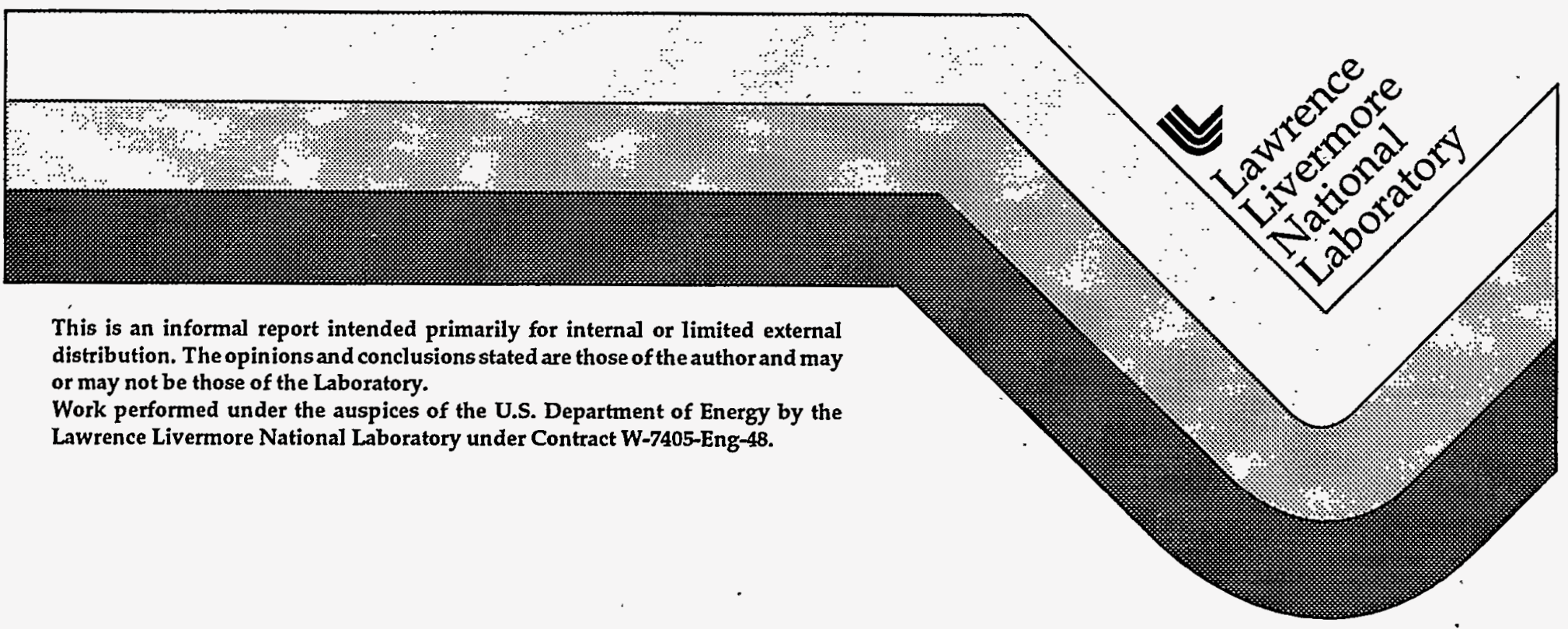




\section{DISCLAIMER}

This document was prepared as an acccount of work sponsored by an agency of the United States Government. Neither the United States Government nor the University of California nor any of their employees, makes any warranty, express or implied, or assumes any legal liability or responsibility for the accuracy, completeness, or usefulness of any information, apparatus, product, or process disclosed, or represents that its use would not infringe privately own rights. Reference herein to any specific commercial products, process, or service by trade name, trademark, manufacturer, or otherwise, does not necessarily constitute or imply its endorsement, recommendation, or favoring by the United States Government or the University of California. The.views and opinions of authors expressed herein do not necessarily state or reflect those of the United States Government or the University of California, and shall not be used for advertising or product endorsement purposes.

This report has been reproduced directly from the best available copy.

Available to DOE and DOE contractors from the Office of Scientific and Technical Information P.O. Box 62, Oak Ridge, IN 37831

Prices available from (615) 576-8401, FIS 626-8401

Available to the public from the

National Technical Information Service

U.S. Department of Commerce 5285 Port Royal Rd.,

Springfield, VA 22161 


\section{DISCLAIMER}

Portions of this document may be illegible in electronic image products. Images are produced from the best available original document. 


\title{
CONJUGATE GRADIENT MATHEW
}

\author{
G. Sugiyama, R. L. Lee, and H. Walker \\ Lawrence Livermore National Laboratory \\ University of California \\ Livermore, Ca. 94550
}

September 20, 1994

\begin{abstract}
MATHEW generates mass-consistent winds by minimal adjustment of input fields derived from observational, analyzed, or model data. The adjustment is performed by a constrained variational minimization using finite-difference methods and a conjugate gradient solution. Generalized symmetric boundary conditions are implemented, which allow control of the relative amounts of normal and tangential adjustment at the lateral and terrain surfaces. We discuss in detail the derivation of the Euler-Lagrange equations, the MATHEW boundary conditions, the finite-difference stencil for the Poisson equation, and the conjugate-gradient algorithm. MATHEW results are then compared with potential flow solutions for simple geometries. We also give examples of the use of boundary conditions to create different kinds of flow fields, to prevent speed reductions, and to eliminate flow artifacts caused by complex terrain. The present MATHEW code is twice as fast as the original successive overrelaxation algorithm and can be straightforwardly generalized from block to continuous terrain representations.
\end{abstract}




\section{Introduction}

The Mass-Adjusted THrEe-dimensional Wind (MATHEW) code builds mass-consistent wind fields, which satisfy the continuity equation for an incompressible fluid, as input for a pollutant transport and diffusion code (ADPIC). Variational techniques are used to minimally adjust input wind fields in order to eliminate sources and sinks and to deal with the strong influences of complex terrain on the flow. The requirement of minimal adjustment maintains consistency with available meteorological measurements (surface and upper air soundings), while atmospheric stability conditions govern the relative amounts of change in the vertical and horizontal wind components. The adjustment is irrotational and neither momentum balance nor energy conservation is imposed.

The underlying theoretical framework for the variational adjustment was developed by Sasaki [1] and applied in the Atmospheric Release Advisory Capability (ARAC) operational context by Sherman [2]. A functional is formulated which minimizes the variance between input and output winds subject to a non-divergence constraint, mass-flow boundary conditions on lateral and top faces, and the requirement of zero normal wind-field components at terrain surfaces. Velocity components are defined on staggered grid faces so that the mass-consistency constraint is cell-flux rather than grid-point based. The problem then reduces to the solution of the Poisson equation in the Lagrange multiplier $\lambda(x, y, z)$, with the adjusted winds derived from the corresponding Euler-Lagrange equations.

The original ARAC MATHEW code discretized the Poisson equation using quadratic fits to generate the boundary conditions, which resulted in an asymmetric coefficient matrix. The equation was then solved by a successive overrelaxation method. In order to implement a more efficient conjugate gradient algorithm and eventually adapt the code to a continuous terrain representation, the problem is reformulated with standard image point boundary conditions. This yields a symmetric matrix representation, which is consistent with the symmetry of the continuous Poisson problem. The boundary conditions are generalized to include parameters for controlling the relative amounts of normal and tangential adjustment at the lateral and top edges (MIXED boundary condition [3] ) and for fixing velocities at the inflow boundaries.

Input wind fields are generally derived by a combination of interpolation and extrapolation from sparse observational data and are typically assumed to be purely horizontal due to the lack of vertical velocity measurements. Such two-dimensional wind fields can 
be prepared for MATHEW using the MEDIC code which incorporates surface, upper air, and analyzed wind data as available and appropriate. However, the mass-adjustment technique is applicable to input winds with a complete set of $u, v$, and $w$ components and can be implemented using three-dimensional wind fields derived from assorted diagnostic and prognostic models.

\section{Calculus of Variations}

A variational problem may be solved by reduction to a set of differential equations known as the Euler equations, which represent necessary, though not sufficient, conditions for a minimum [4]. For MATHEW, the integral $J$ that we wish to minimize is a function of the wind-field components and their first derivatives in a simulation box of volume $\Omega$

$$
J[u, v, w]=\int_{\Omega} F\left(x, y, z, u, v, w, \dot{u}_{x}, u_{y}, \dot{u}_{z}, v_{x}, v_{y}, v_{z}, \dot{w}_{x}, w_{y}, w_{z}\right) d x d y d z
$$

We introduce three arbitrary functions, $\eta(x, y, z), \zeta(x, y, z)$, and $\xi(x, y, z)$, and construct the functions $u=\tilde{u}+\epsilon_{\eta} \eta \equiv \tilde{u}+\delta u, v=\tilde{v}+\epsilon_{\zeta} \zeta \equiv \tilde{v}+\delta v$, and $w=\tilde{w}+\epsilon_{\xi} \xi \equiv \tilde{w}+\delta w$, where $\epsilon_{\eta}, \epsilon_{\zeta}$, and $\epsilon_{\xi}$ are parameters, $\tilde{u}(x, y, z), \tilde{v}(x, y, z)$ and $\tilde{w}(x, y, z)$ are the desired extremal functions, and $\delta u, \delta v, \delta w$ are the variations in the $u, v$, and $w$ components, respectively. An extremum value of the integral $J[u, v, w]$ occurs when all three $\epsilon$ 's vanish or equivalently when the derivative of $J$ with respect to each of the $\epsilon$ 's is zero. This condition may be written compactly in terms of the first variation of $J$ as

$$
\begin{aligned}
0=\delta J & =\epsilon_{\eta}\left(\frac{d}{d \epsilon_{\eta}} J\left[\tilde{u}+\epsilon_{\eta} \eta, \tilde{v}+\epsilon_{\zeta} \zeta, \tilde{w}+\epsilon_{\xi} \xi, \tilde{u}_{x}+\epsilon_{\eta} \eta_{x}, \tilde{u}_{y}+\epsilon_{\eta} \eta_{y}, \tilde{u}_{z}+\epsilon_{\eta} \eta_{z}, \cdots\right]\right)_{\epsilon_{\eta}=0} \\
& +\epsilon_{\zeta}\left(\frac{d J}{d \epsilon_{\zeta}}\right)_{\epsilon_{\zeta}=0}+\epsilon_{\xi}\left(\frac{d J}{d \epsilon_{\xi}}\right)_{\epsilon_{\xi}=0}
\end{aligned}
$$

Differentiating with respect to the $\epsilon$ 's under the integral sign, integrating by parts, and separating into volume and surface integrals yields

$$
\begin{aligned}
0=\delta J= & \epsilon_{\eta} \int_{\Omega}\left(\eta \frac{\partial F}{\partial u}+\frac{\partial F}{\partial u_{x}} \eta_{x}+\frac{\partial F}{\partial u_{y}} \eta_{y}+\frac{\partial F}{\partial u_{z}} \eta_{z}\right)+\epsilon_{\zeta} \int_{\Omega} \cdots+\epsilon_{\xi} \int_{\Omega} \cdots \\
= & \epsilon_{\eta} \int_{\Omega} \eta\left(\frac{\partial F}{\partial u}-\frac{\partial F}{\partial u_{x}}-\frac{\partial F}{\partial u_{y}}-\frac{\partial F}{\partial u_{z}}\right) d x d y d z \\
& \quad+\epsilon_{\eta} \int_{\Omega} \frac{d}{d x}\left(\eta \frac{\partial F}{\partial u_{x}}\right)+\frac{d}{d y}\left(\eta \frac{\partial F}{\partial u_{y}}\right)+\frac{d}{d z}\left(\eta \frac{\partial F}{\partial u_{z}}\right) d x d y d z \\
\quad & \quad+\epsilon_{\zeta} \int_{\Omega} \cdots+\epsilon_{\xi} \int_{\Omega} \cdots \\
= & \int_{\Omega} \delta u\left(\frac{\partial F}{\partial u}-\frac{\partial F}{\partial u_{x}}-\frac{\partial F}{\partial u_{y}}-\frac{\partial F}{\partial u_{z}}\right)+\int_{\Omega} \delta v \cdots+\int_{\Omega} \delta w \cdots \\
& \quad+\int_{\mathcal{S}} \delta u\left(\frac{\partial F}{\partial u_{x}} n_{x}+\frac{\partial F}{\partial u_{y}} n_{y}+\frac{\partial F}{\partial u_{z}} n_{z}\right)+\int_{\mathcal{S}} \delta v \cdots+\int_{\mathcal{S}} \delta w \cdots,
\end{aligned}
$$


where $u_{x}, u_{y}$, and $u_{z}$ are the derivatives of $u$ with respect to $x, y$, and $z$, respectively, $n_{x}$, $n_{y}$, and $n_{z}$ are the components of the unit normal to the surface $\mathcal{S}$, and the ellipses indicate the corresponding terms in $v-\zeta$ and $w-\xi$. Then, by the fundamental lemma of the calculus of variations, the volume integrands must vanish, yielding the Euler equations

$$
\begin{gathered}
\frac{\partial F}{\partial u}-\frac{d}{d x}\left(\frac{\partial F}{\partial u_{x}}\right)-\frac{d}{d y}\left(\frac{\partial F}{\partial u_{y}}\right)-\frac{d}{d z}\left(\frac{\partial F}{\partial u_{z}}\right)=0 \\
\frac{\partial F}{\partial v}-\frac{d}{d x}\left(\frac{\partial F}{\partial v_{x}}\right)-\frac{d}{d y}\left(\frac{\partial F}{\partial v_{y}}\right)-\frac{d}{d z}\left(\frac{\partial F}{\partial v_{z}}\right)=0 \\
\frac{\partial F}{\partial \dot{w}}-\frac{d}{d x}\left(\frac{\partial F}{\partial w_{x}}\right)-\frac{d}{d y}\left(\frac{\partial F}{\partial w_{y}}\right)-\frac{d}{d z}\left(\frac{\partial F}{\partial w_{z}}\right)=0
\end{gathered}
$$

In the case of prescribed boundary values, the wind components are fixed on $\mathcal{S}$, and the surface integrals do not contribute to $\delta J$. Generally, however, the variations $\delta u, \delta v$, and $\delta w$ are free to assume arbitrary values on the surface. This leads to a set of free or natural boundary condition equations

$$
\begin{aligned}
\left(\frac{\partial F}{\partial u_{x}} n_{x}+\frac{\partial F}{\partial u_{y}} n_{y}+\frac{\partial F}{\partial u_{z}} n_{z}\right) \delta u & =0 \\
\left(\frac{\partial F}{\partial v_{x}} n_{x}+\frac{\partial F}{\partial v_{y}} n_{y}+\frac{\partial F}{\partial v_{z}} n_{z}\right) \delta v & =0 \\
\left(\frac{\partial F}{\partial w_{x}} n_{x}+\frac{\partial F}{\partial w_{y}} n_{y}+\frac{\partial F}{\partial w_{z}} n_{z}\right) \delta w & =0
\end{aligned}
$$

which must also be satisfied by an extremum solution. Addition of either volume integrand divergence expressions or surface integral terms to the functional modifies the boundary conditions without altering the Euler equations.

Subsidiary conditions or constraints can be imposed on the fields $u, v$, and $w$ through the use of Lagrange multipliers. In general, for any constraint that can be expressed in the form

$$
G\left(x, y, z, u, v, w, u_{x}, u_{y}, u_{z}, v_{x}, v_{y}, v_{z}, w_{x}, w_{y}, w_{z}\right)=0
$$

the variational problem can be solved by forming the differential equations for the integrand $F+\lambda G$, for a suitable value of the Lagrange multiplier $\lambda$. The resulting Euler-Lagrange differential equations together with the constraint are sufficient to determine both the extremal solution and the value of $\lambda$. 
In its simplest form, the MATHEW functional required to minimize the variance between the observed input and adjusted output winds subject to the mass-consistency constraint is

$J(u, v, w, \lambda)=\int_{\Omega} \frac{1}{2 \sigma_{H}^{2}}\left(\left(u-u^{\circ}\right)^{2}+\left(v-v^{\circ}\right)^{2}\right)+\frac{1}{2 \sigma_{V}^{2}}\left(w-w^{\circ}\right)^{2}+\lambda\left(\frac{\partial u}{\partial x}+\frac{\partial v}{\partial y}+\frac{\partial w}{\partial z}\right) d x d y d z$

where $(u, v, w)$ and $\left(u^{o}, v^{o}, w^{o}\right)$ are the output and input wind components, respectively, $\lambda(x, y, z)$ is the Lagrange multiplier for the mass-consistency constraint, $\Omega$ is the volume domain of the simulation, and $\sigma_{V}$ and $\sigma_{H}$ are parameters controlling the relative amounts of vertical and horizontal adjustment. From Eqs. 4 and 5, the Euler-Lagrange equations associated with this functional are

$$
\begin{aligned}
u-u^{o} & =\sigma_{H}^{2} \frac{\partial \lambda}{\partial x} \\
v-v^{o} & =\sigma_{H}^{2} \frac{\partial \lambda}{\partial y} \\
w-w^{o} & =\sigma_{V}^{2} \frac{\partial \lambda}{\partial z}
\end{aligned}
$$

with boundary conditions

$$
\begin{aligned}
& \left.\lambda n_{x} \delta u\right|_{\mathcal{S}}=0 \\
& \left.\lambda n_{y} \delta v\right|_{\mathcal{S}}=0 \\
& \left.\lambda n_{z} \delta w\right|_{\mathcal{S}}=0 .
\end{aligned}
$$

Taking appropriate derivatives of the Euler-Lagrange equations and substituting into the mass-consistency constraint yields the Poisson equation

$$
\sigma_{H}^{2}\left(\frac{\partial^{2} \lambda}{\partial x^{2}}+\frac{\partial^{2} \lambda}{\partial y^{2}}\right)+\sigma_{v}^{2} \frac{\partial^{2} \lambda}{\partial z^{2}}=-\frac{\partial u^{o}}{\partial x}-\frac{\partial v^{\circ}}{\partial y}-\frac{\partial w^{\circ}}{\partial z}
$$

which may be solved for $\lambda$ subject to the boundary conditions. The adjusted wind fields are then determined from Eqs. 8.

\section{Boundary Conditions}

The boundary conditions of Eqs: 9 can be satisfied if $\left.\lambda\right|_{s}=0$. The physical interpretation of this solution is revealed by examination of the Euler-Lagrange equations. If $\lambda$ is a 
constant on $\mathcal{S}$, the derivatives along the surface vanish, which fixes the tangential component of the velocity, while imposing no restrictions on the normal component. This is the ADJUST_NORMAL condition and is a kind of flow-through boundary. Eqs. 9 are also satisfied if $\left.\frac{\partial \lambda}{\partial n}\right|_{\mathcal{S}}=0$, which prevents changes in the normal velocity component, while allowing free adjustment of the tangential component. This is a FIXED_NORMAL or fixed-flow boundary and can be used to maintain zero normal velocities on terrain surfaces ${ }^{1}$.

It is advantageous to incorporate a new surface integral term which provides more flexibility in the boundary conditions and to treat the FIXED_NORMAL case in a constraint formalism, so that input wind fields with non-zero normal components at terrain surfaces can be used. These considerations lead to a generalized MATHEW functional .

$$
\begin{aligned}
J(u, v, w, \lambda) & =\int_{\Omega}\left[\left(\vec{u}-\vec{u}^{\circ}\right) \cdot \mathbf{D}^{-1} \cdot\left(\vec{u}-\vec{u}^{o}\right)+\lambda \nabla \cdot \vec{u}\right] d x d y d z \\
& -\int_{\mathcal{S}_{\text {terrain }}} \lambda_{\text {terrain }} \vec{n} \cdot \vec{u} d \mathcal{S} \\
& +\frac{1}{2} \int_{\mathcal{S}_{\text {flow }}} f(\mathcal{S})\left(\vec{u}-\vec{u}^{o}\right)^{2} d \mathcal{S}
\end{aligned}
$$

where $f(\mathcal{S})$ is an arbitrary continuous function on the flow-through boundary $\mathcal{S}_{\text {flow }}, \mathcal{S}_{\text {terrain }}$ is the terrain surface, and $\mathbf{D}$ is a $3 \times 3$ diagonal matrix with non-zero components $\sigma_{i}^{2} \delta_{i, j}\left(\delta_{i, j}\right.$ is the Kronecker delta and $\sigma_{1}=\sigma_{2}=\cdot \sigma_{H}$ and $\sigma_{3}=\sigma_{V}$ ).

The Euler-Lagrange and Poisson equations are unchanged from the simple MATHEW formulation (Eqs. 8 and 10)

$$
\begin{gathered}
\vec{u}-\vec{u}^{o}=\mathbf{D} \cdot \nabla \lambda \\
\nabla \cdot \mathbf{D} \cdot \nabla \lambda=-\nabla \cdot \vec{u}^{\circ},
\end{gathered}
$$

but the boundary conditions now contain contributions from the new surface integrals as well as from the integration by parts

$$
\begin{aligned}
0 & =\int_{\mathcal{S}} \lambda \vec{n} \cdot \delta \vec{u} d \mathcal{S}-\int_{\mathcal{S}_{\text {terrain }}} \lambda_{\text {terrain }} \vec{n} \cdot \delta \vec{u} d \mathcal{S}+\int_{\mathcal{S}_{\text {flow }}} f(\mathcal{S})\left(\vec{u}-\vec{u}^{\circ}\right) \cdot \delta \vec{u} d \mathcal{S} \\
& =\int_{\mathcal{S}_{\text {terrain }}}\left(\lambda_{\text {terrain }}-\lambda\right) \vec{n} \cdot \delta \vec{u} d \mathcal{S}+\int_{\mathcal{S}_{\text {flow }}}\left[f(\mathcal{S})\left(\vec{u}-\vec{u}^{o}\right)+\lambda \vec{n}\right] \cdot \delta \vec{u} d \mathcal{S}
\end{aligned}
$$

For the first integral to vanish, $\lambda$ must be equal to $\lambda_{\text {terrain }}$ on $\mathcal{S}_{\text {terrain }}$. The flow-through boundary condition and terrain surface constraint can then be written as

$$
\begin{array}{ll}
\lambda+f(\mathcal{S}) \vec{n} \cdot\left(\vec{u}-\vec{u}^{o}\right)=\lambda+f(\mathcal{S}) \vec{n} \cdot \mathbf{D} \cdot \nabla \lambda=0 & \mathcal{S}_{\text {flow }} \\
\vec{n} \cdot \vec{u}=\vec{n} \cdot \vec{u}^{o}+\vec{n} \cdot \mathbf{D} \cdot \nabla \lambda=\mathbf{0} & \mathcal{S}_{\text {terrain }}
\end{array}
$$

\footnotetext{
${ }^{1}$ The original MATHEW had OPEN boundaries corresponding to the ADJUST_NORMAL condition and CLOSED boundaries equivalent to FIXED_NORMAL on terrain surfaces with $\vec{n} \cdot \vec{u} \mid s_{\text {terrain }}=0$.
} 


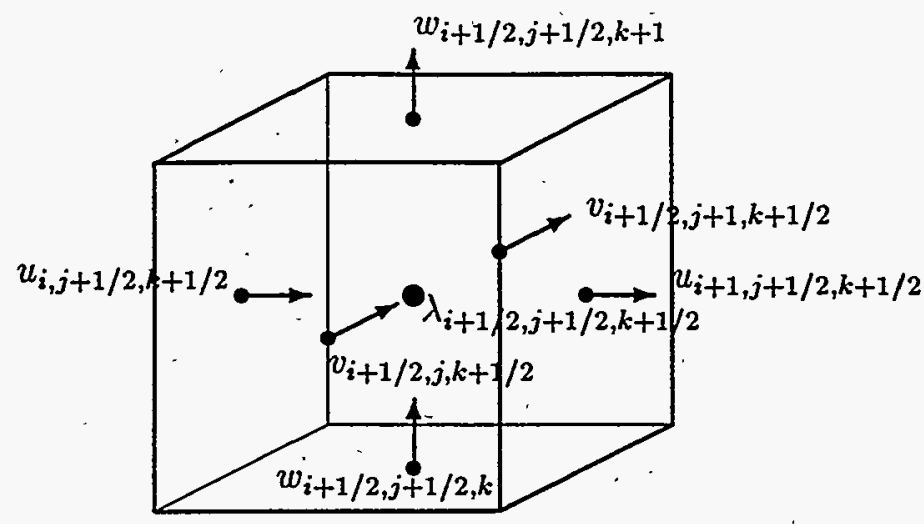

Figure 1: Schematic representation of MATHEW cell centered at point $(i+1 / 2, j+1 / 2, k+1 / 2)$ showing staggered face-centered wind and cell-centered $\lambda$ components.

where the Euler-Lagrange equations have been used to substitute for $\vec{u}$. For a rectangular grid, these equations reduce to

$$
\begin{array}{ll}
\lambda+\sigma_{n}^{2} f(\mathcal{S}) \frac{\partial \lambda}{\partial n}=0 & \mathcal{S}_{\text {flow }} \\
\vec{n} \cdot \vec{u}^{\circ}=-\sigma_{n}^{2} \frac{\partial \lambda}{\partial n} & \mathcal{S}_{\text {terrain }}
\end{array}
$$

where $\vec{n}$ is the outward normal to the face. Although in principle, $f(\mathcal{S})$ could be any continuous function, in practice, it is taken to be a constant $1 / c_{n}$ governing the ratio of normal to tangential adjustment on each face, in order to limit the danger of overly constraining the problem. The ADJUST_NORMAL and FIXED_NORMAL boundary conditions are limiting cases of the MIXED boundary condition as $c_{n} \rightarrow \infty$ or $c_{n} \rightarrow 0$, respectively.

\section{Derivation of the MATHEW stencil .}

The MATHEW grid is rectangular with regular spacings of $\Delta x, \Delta y$, and $\Delta z$, indexed by $i, j$ and $k$, which run from 1 to $i_{\max }, j_{\max }$, and $k_{\max }$, respectively. The wind field components are defined on staggered face centers, interpolated from input grid-point winds, and $\lambda$ is defined at the cell center as shown in Figure 1. Standard two- and three-point finite difference formulae then give the first derivatives of $\lambda$ on the staggered face centers and both 


\begin{tabular}{|l|l|l||l|}
\hline CELL $i+\frac{1}{2}$ & BOUNDARY $i$ & BOUNDARY $i+1$ & \\
\hline \hline terrain & & & $\lambda_{i+1 / 2}=1$ \\
\hline interior & FIXED_NORMAL & & $\lambda_{i-1 / 2}=\lambda_{i+1 / 2}$ \\
& & FIXED_NORMAL & $\lambda_{i+3 / 2}=\lambda_{i+1 / 2}$ \\
& \multirow{4}{*}{ ADJUST_NORMAL } & & $\lambda_{i-1 / 2}=-\lambda_{i+1 / 2}$ \\
& & ADJUST_NORMAL & $\lambda_{i+3 / 2}=-\lambda_{i+1 / 2}$ \\
& & & $\lambda_{i-1 / 2}=\left(\frac{1-c_{x}^{-} \frac{\Delta x}{2}}{1+c_{x}^{-} \frac{\Delta x}{2}}\right) \lambda_{i+1 / 2}$ \\
& MIXED & & $\lambda_{i+3 / 2}=\left(\frac{1-c_{x}^{+} \frac{\Delta x}{2}}{1+c_{x}^{+} \frac{\Delta x}{2}}\right) \lambda_{i+1 / 2}$ \\
\hline
\end{tabular}

Table 1: Boundary condition restrictions on $\lambda$ values. The constant $c_{x}^{ \pm}$controls the ratio of normal to horizontal adjustment for the MIXED condition.

the second $\lambda$ derivative and the wind component first derivatives at the cell centers. The topography is specified by cell-centered heights indexed by $\left(i+\frac{1}{2}, j+\frac{1}{2}\right)$.

For computational purposes, the Poisson equation is divided through by $\sigma_{H}^{2}$ and discretized, resulting in a set of linear equations in the components $\lambda_{i+\frac{1}{2}, j+\frac{1}{2}, k+\frac{1}{2}}$, which can be written in matrix form as $\mathbf{C} \vec{\lambda}=\overrightarrow{\mathrm{b}} . \quad \vec{\lambda}$ and $\vec{b}=-\left(\nabla \cdot \vec{u}_{o}\right) / \sigma_{H}^{2}$ are vectors of length $N=\left(i_{\max }-1\right) \cdot\left(j_{\max }-1\right) \cdot\left(k_{\max }-1\right)$, and $\mathrm{C}$ is a $N \mathrm{x} N$ matrix containing the finitedifference coefficients for each cell, which depend upon the boundary conditions. Since the upper boundary of a given cell is the lower boundary of the next higher index cell on that axis, $\mathrm{C}$ is symmetrical, and can be constructed to be positive definite $(\vec{a} \cdot \mathbf{C} \cdot \vec{a} \geq 0$ for all vectors $\vec{a})^{2}$.

The stencil for the coefficient matrix can be derived for any one of the three axis directions, with symmetry used to establish the other components. In the $x$ direction, the boundary conditions on the $i$ and $i+1$ faces determine relationships between $\lambda_{i-1 / 2}, \lambda_{i+1 / 2}$, and $\lambda_{i+3 / 2}$ as summarized in Table 1 . Terrain cells are decoupled by setting the diagonal $\lambda_{i+1 / 2}$ element to one and the off-diagonal elements of the matrix row to zero, so that the value of $\lambda$ for a terrain cell is forced to be zero. Bordering interior cells impose no constraints

\footnotetext{
${ }^{2}$ The original MATHEW stencil was derived using quadratic differencing formulae leading to an asymmetric coefficient matrix.
} 


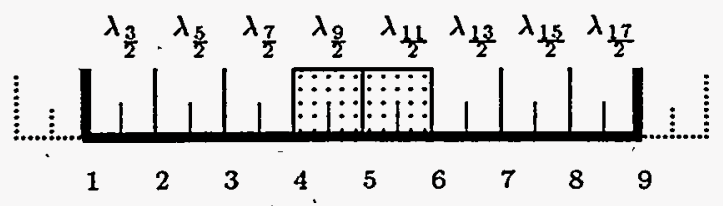

Figure 2: One-dimensional example. The heavy line represents the simulation box and the filled boxed represent terrain cells.

on the $\lambda$ values. Other boundary conditions restrict the allowed values of the $\lambda$ components and are used to modify the discretized one-dimensional Poisson and Euler-Lagrange equations

$$
\frac{\lambda_{i+3 / 2}-2 \lambda_{i+1 / 2}+\lambda_{i-1 / 2}}{(\Delta x)^{2}}=-\frac{1}{\sigma_{H}^{2}} \frac{u_{i+1}^{o}+u_{i}^{o}}{\Delta x}, \quad u_{i}=u_{i}^{o}+\frac{\lambda_{i+1 / 2}-\lambda_{i-1 / 2}}{\Delta x}
$$

as illustrated in Table 2. For ADJUST_NORMAL boundary conditions, examination shows that the tangential component is algebraically constrained but not fixed, due to the staggered face-centered representation of the wind components. The opposite sign on the derivative for the $i$ MIXED boundary condition reflects the reverse direction of the outward normal.

Combinations of these boundary conditions can be solved in the same manner. As an example, consider the one dimensional system of Fig. 2. The simulation box is delimited by the heavy line and contains 8 cells. The $\lambda$ values are located at the centers indexed by $i+\frac{1}{2}, i: 1 \rightarrow 8$ and the wind components $\vec{u}$ and $\vec{u}_{i}^{\circ}$ are defined on the 9 cell faces. The input wind components on and inside the terrain are zero. The Poisson matrix equation associated with this problem is

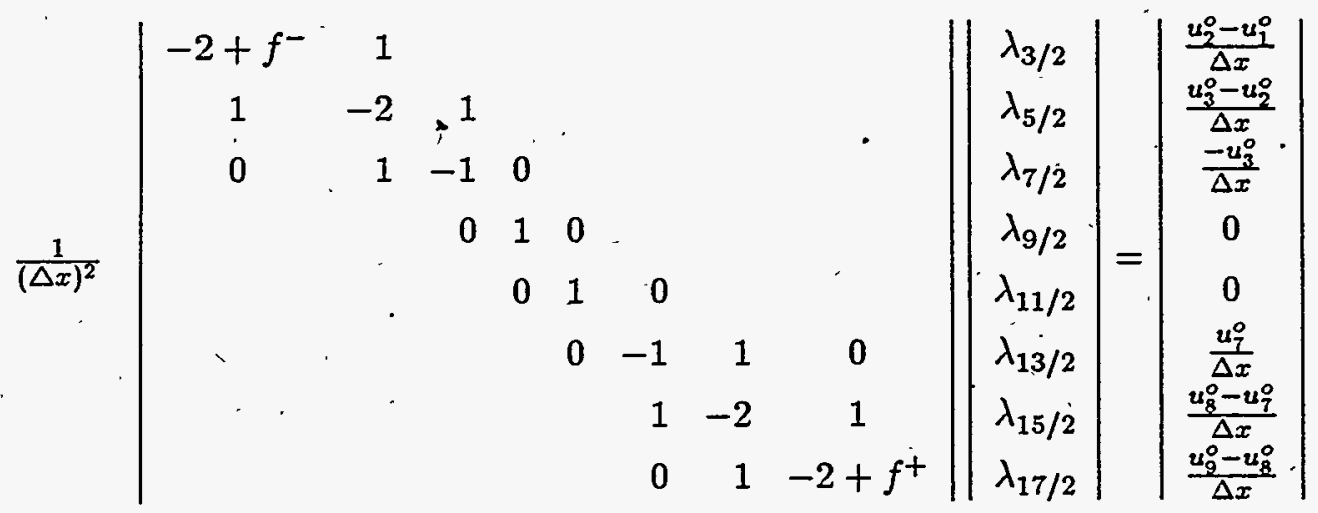




\begin{tabular}{|c|c|c|}
\hline$\lambda_{i}=0$ & $\lambda_{i+1}=0$ \\
\hline$\left.\frac{\partial \lambda}{\partial x}\right|_{i}$ & $\frac{2 \lambda_{i+1 / 2}}{\Delta x}$ & $\left.\frac{\partial \lambda}{\partial x}\right|_{i}=\frac{\lambda_{i+1 / 2}-\lambda_{i-1 / 2}}{\Delta x}$ \\
$\left.\frac{\partial \lambda}{\partial x}\right|_{i+1}$ & $=\frac{\lambda_{i+3 / 2}-\lambda_{i+1 / 2}}{\Delta x}$ & $\left.\frac{\partial \lambda}{\partial x}\right|_{i+1}=-\frac{2 \lambda_{i+1 / 2}}{\Delta x}$ \\
$\left.\frac{\partial^{2} \lambda}{\partial x^{2}}\right|_{i+1 / 2}=\frac{\lambda_{i+3 / 2}-3 \lambda_{i+1 / 2}}{(\Delta x)^{2}}$ & $\left.\frac{\partial^{2} \lambda}{\partial x^{2}}\right|_{i+1 / 2}=\frac{-3 \lambda_{i+1 / 2}+\lambda_{i-1 / 2}}{(\Delta x)^{2}}$
\end{tabular}

\begin{tabular}{|c|c|c|c|c|}
\hline$\left.\frac{\partial \lambda}{\partial x}\right|_{i}$ & $=0$ & $\left.\frac{\partial \lambda}{\partial x}\right|_{i+1}$ & & 0 \\
\hline $\begin{array}{l}\lambda_{i+1 / 2} \\
\left.\frac{\partial \lambda}{\partial x}\right|_{i+1} \\
\left.\frac{\partial^{2} \lambda}{\partial x^{2}}\right|_{i+1 / 2}\end{array}$ & $\begin{array}{l}=\lambda_{i-1 / 2} \\
=\frac{\lambda_{i+3 / 2}-\lambda_{i+1 / 2}}{\Delta x} \\
=\frac{\lambda_{i+3 / 2}-\lambda_{i+1 / 2}}{(\Delta x)^{2}}\end{array}$ & $\begin{array}{l}\lambda_{i+3 / 2} \\
\left.\frac{\partial \lambda}{\partial x}\right|_{i} \\
\left.\frac{\partial^{2} \lambda}{\partial x^{2}}\right|_{i+1 / 2}\end{array}$ & $\begin{array}{l}= \\
= \\
=\end{array}$ & $\begin{array}{l}\lambda_{i+1 / 2} \\
\frac{\lambda_{i+1 / 2}-\lambda_{i-1 / 2}}{\Delta x} \\
\frac{-\lambda_{i+1 / 2}+\lambda_{i-1 / 2}}{(\Delta x)^{2}}\end{array}$ \\
\hline
\end{tabular}

\begin{tabular}{|c|c|c|c|}
\hline$\left.\left(-\frac{\partial \lambda}{\partial x}+\lambda\right)\right|_{i}=0$ & $\left.\left(\frac{\partial \lambda}{\partial x}+\lambda\right)\right|_{i+1}=0$ \\
\hline$\left.\frac{\partial \lambda}{\partial x}\right|_{i}$ & $=\frac{\lambda_{i+1 / 2}-\lambda_{i-1 / 2}}{\Delta x}$ \\
$\left.\frac{\partial \lambda}{\partial x}\right|_{i+1}=\frac{\lambda_{i+3 / 2}-\lambda_{i+1 / 2}}{\Delta x}$ & $\left.\frac{\partial \lambda}{\partial x}\right|_{i}$ & $=\left(\frac{1-\frac{\Delta x}{2}}{1+\frac{\Delta x}{2}}-1\right) \frac{\lambda_{i+1 / 2}}{\Delta x}$ \\
$\left.\frac{\partial^{2} \lambda}{\partial x^{2}}\right|_{i+1 / 2}=\left.\frac{\lambda_{i+1 / 2}}{(\Delta x)^{2}}\right|_{i+1}$ & $=\frac{\lambda_{i-1 / 2}}{(\Delta x)^{2}}$ \\
$+\frac{\lambda_{i+1 / 2}}{(\Delta x)^{2}}\left(-2+\frac{1-\frac{\Delta x}{2}}{1+\frac{\Delta x}{2}}\right)$ & $\begin{array}{r}\left.\frac{\partial^{2} \lambda}{\partial x^{2}}\right|_{i+1 / 2} \\
(\Delta x)^{2}\end{array}$
\end{tabular}

Table 2: Discretized equations for (a) ADJUST_NORMAL, (b) FIXED_NORMAL, and (c) MIXED boundary conditions. 
where all unspecified elements are zero and

$$
f^{ \pm}= \begin{cases}-1 & \lambda=0 \\ +1 & \frac{\partial \lambda}{\partial x}=0 \\ \frac{1-c_{x}^{ \pm} \Delta x / 2}{1+c_{x}^{ \pm} \Delta x / 2} & \frac{\partial \lambda}{\partial x} \pm c_{x}^{ \pm} \lambda=0\end{cases}
$$

depending on the lateral boundary conditions. Once this equation is solved for the $\lambda_{i+1 / 2}$, the adjusted winds can be found from the discretized Euler-Lagrange equations

$$
\left|\begin{array}{l}
u_{1} \\
u_{2} \\
u_{3} \\
u_{4} \\
u_{5} \\
u_{6} \\
u_{7} \\
u_{8} \\
u_{9}
\end{array}\right|=\left|\begin{array}{l}
u_{1}^{o} \\
u_{2}^{o} \\
u_{3}^{o} \\
0 \\
0 \\
0 \\
u_{7}^{o} \\
u_{8}^{o} \\
u_{9}^{o}
\end{array}\right|+\left|\begin{array}{l}
\sigma_{H}^{2} g^{-} \frac{\lambda_{3 / 2}}{\Delta x} \\
\sigma_{H}^{2} \frac{\lambda_{5 / 2}-\lambda_{3 / 2}}{\Delta x} \\
\sigma_{H}^{2} \frac{\lambda_{7 / 2}-\lambda_{5 / 2}}{\Delta x} \\
0 \\
0 \\
0 \\
\sigma_{H}^{2} \frac{\lambda_{15 / 2}-\lambda_{13 / 2}}{\Delta x} \\
\sigma_{H}^{2} \frac{\lambda_{17 / 2}-\lambda_{15 / 2}}{\Delta x} \\
\sigma_{H}^{2} g^{+} \frac{\lambda_{17 / 2}}{\Delta x}
\end{array}\right|
$$

where

$$
g^{ \pm}=\left\{\begin{array}{ll}
\mp 2 & \lambda=0 \\
0 & \frac{\partial \lambda}{\partial x}=0 \\
\mp \frac{c_{x}^{ \pm} \Delta x}{1+c_{x}^{ \pm} \Delta x / 2} & \frac{\partial \lambda}{\partial x} \pm c_{x}^{ \pm} \lambda=0
\end{array} .\right.
$$

corresponding to the lateral boundary conditions in $f^{ \pm}$.

Due to the.symmetric sparse nature of the Poisson matrix, considerable memory savings are possible by storing only the diagonal and sub-diagonal elements. For the threedimensional case, this can be generalized to storing the four possibly non-zero lower triangle components of $\mathbf{C}$ in a compact $4 \times \mathrm{x}$ form written schematically as

$$
\mathrm{A}=\left|\begin{array}{llll}
\vdots & \vdots & \vdots & \vdots \\
C_{i, j, k-1} & C_{i, j-1, k} & C_{i-1, j, k} & C_{i, j, k} \\
\vdots & \vdots & \vdots & \vdots
\end{array}\right|
$$

where $N$ is the number of cells. The $n^{\text {th }}$ or $(i, j, k)^{\text {th }}$ element of the matrix multiply $\mathrm{C} \cdot \lambda$ 
then becomes

$$
\begin{aligned}
(\mathrm{C} \cdot \vec{\lambda})_{n} & =(\mathrm{C} \cdot \vec{\lambda})_{i, j, k} \\
& =A_{i, j, k, 4} \lambda_{i, j, k}+A_{i, j, k, 3} \lambda_{i-1, j, k}+A_{i, j, k, 2} \lambda_{i, j-1, k}+A_{i, j, k, 1} \lambda_{i, j, k-1} \\
& +A_{i+1, j, k, 3} \lambda_{i+1, j, k}+A_{i, j+1, k, 2} \lambda_{i, j+1, k}+A_{i, j, k+1,1} \lambda_{i, j, k+1} \\
& =A_{n, 4} \lambda_{n}+A_{n, 3} \lambda_{n-1}+A_{n, 2} \lambda_{n-i_{\max }}+A_{n, 1} \lambda_{n-i_{\max } \cdot j_{\max }} \\
& +A_{n+1,3} \lambda_{n+1}+A_{n+i_{\max }, 2} \lambda_{n+i_{\max }}+A_{n+i_{\max } \cdot j_{\max , 1}} \lambda_{n+i_{\max } \cdot j_{\max }}
\end{aligned}
$$

since $\pm i_{\max }$ moves \pm 1 in the $j$ coefficient and $\pm\left(i_{\max } * j_{\max }\right)$ moves \pm 1 in the $k$ coefficient. We will also call this the matrix multiply $\mathbf{A} \cdot \lambda$.

The complete MATHEW stencil in three-dimensions is given in terms of the components of $\mathbf{A}$ in Table 3. The matrix elements are found by summing together the contributions in each column which match the appropriate boundary conditions. For example, if the cell $n=(i, j, k)$ is bordered by terrain cells at $i-1$ and $j+1$ and by interior cells on the remaining sides, $A_{n, 1}=\left(\frac{\sigma_{V}}{\sigma_{H}}\right)^{2} \frac{1}{(\Delta z)^{2}}, A_{n, 2}=\frac{1}{(\Delta y)^{2}}, A_{n, 3}=0$, and $A_{n, 4}=-\frac{1}{(\Delta x)^{2}}-$ $\frac{1}{(\Delta y)^{2}}-\left(\frac{\sigma_{V}}{\sigma_{H}}\right)^{2} \frac{2}{(\Delta z)^{2}}$.

\section{Conjugate Gradient Algorithm}

Conjugate gradient methods for solving an $N \times N$ linear system $\mathbf{A} \vec{x}=\vec{b}$ have several advantageous features. They require no parameter estimations and provide optimal error minimization. The algorithms have a finite termination after $M \leq N$ steps, where $M$ is the number of distinct eigenvalues of $\mathbf{A}$. Since they reference $\mathbf{A}$ only through vector multiplication, the codes are also highly vectorizable and allow concise storage for sparse systems.

Conjugate gradient algorithms [5] are based on the idea of minimizing a function $f(\vec{x})=$ $\frac{1}{2} \vec{x}^{T} \cdot \mathbf{A} \cdot \vec{x}-\vec{x}^{T} \cdot \vec{b}$ by finding the point where $\nabla f=\mathbf{A} \cdot \vec{x}-\vec{b}=0$. The minimization is carried out by generating a succession of search directions $\vec{p}_{k}$ and a corresponding set of improved solutions $\vec{x}_{k+1}$ which minimize $f$ in the space spanned by $\left\{\vec{p}_{1}, \vec{p}_{2}, \cdots, \vec{p}_{k}\right\}$. Convergence is monotonic, the generalized error function $(\mathbf{A} \vec{x}-\vec{z})^{T} \cdot(\vec{x}-\vec{z})$ taking on its smallest value in the subspace spanned by the search when $\vec{z}=\vec{x}_{k+1}$ and decreasing in magnitude with each iteration. 


\begin{tabular}{|c|c|c|c|c|c|c|}
\hline CELL & \multicolumn{2}{|c|}{ BOUNDARY } & $A_{n, 1}$ & $A_{n, 2}$ & $A_{n, 3}$ & $A_{n, 4}$ \\
\hline terrain & \multicolumn{2}{|c|}{ all boundaries } & & & & 1 \\
\hline \multirow[t]{25}{*}{ interior } & \multicolumn{2}{|c|}{ all boundaries } & & & & $-\frac{2}{(\Delta x)^{2}}-\frac{2}{(\Delta y)^{2}}-\left(\frac{\sigma_{Y}}{\sigma_{H}}\right)^{2} \frac{2}{(\Delta z)^{2}}$ \\
\hline & \multirow[t]{4}{*}{$x_{i-1}$} & interior & & & $\frac{1}{(\Delta x)^{2}}$ & \\
\hline & & $\lambda=0$ & & & & $-\frac{1}{(\Delta x)^{2}}$ \\
\hline & & $\frac{\partial \lambda}{\partial x}=0$ & & & & $\frac{1}{(\Delta x)^{2}}$ \\
\hline & & $c_{x}^{-} \lambda-\frac{\partial \lambda}{\partial x}=0$ & - & & - & $\frac{1-c_{x}^{c} \Delta x / 2}{1+c_{x}^{c} \Delta x / 2} \cdot \frac{1}{(\Delta x)^{2}}$ \\
\hline & \multirow[t]{4}{*}{$x_{i+1}$} & interior & & & & \\
\hline & & $\lambda=0$ & & . & & $-\frac{1}{(\Delta x)^{2}}$ \\
\hline & & $\frac{\partial \lambda}{\partial x}=0$ & & & & $\frac{1}{(\Delta x)^{2}}$ \\
\hline & & $c_{x}^{+} \lambda+\frac{\partial \lambda}{\partial x}=0$ & & & & $\frac{1-c_{x}^{+} \Delta x / 2}{1+c_{x}^{+} \Delta x / 2} \cdot \frac{1}{(\Delta x)^{2}}$ \\
\hline & \multirow[t]{4}{*}{$y_{j-1}$} & interior & & $\frac{1}{(\Delta y)^{2}}$ & & \\
\hline & & $\lambda=0$ & & $\cdot$ & & $-\frac{1}{(\Delta y)^{2}}$ \\
\hline & & $\frac{\partial \lambda}{\partial y}=0$ & & & & $\frac{1}{(\Delta y)^{2}}$ \\
\hline & & $c_{y}^{-} \lambda-\frac{\partial \lambda}{\partial y}=0$ & & & & $\frac{1-c_{y}^{-} \Delta y / 2}{1+c_{y}^{-} \Delta y / 2} \cdot \frac{1}{(\Delta y)^{2}}$ \\
\hline & \multirow{4}{*}{$y_{j+1}$} & interior & & & & \\
\hline & & $\lambda=0$ & & & & $-\frac{1}{(\Delta y)^{2}}$ \\
\hline & & $\frac{\partial \lambda}{\partial y}=0$ & & & & $\frac{1}{(\Delta y)^{2}}$ \\
\hline & & $c_{y}^{+} \lambda+\frac{\partial \lambda}{\partial y}=0$ & & & 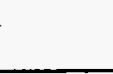 & $\frac{1-c_{y}^{+} \Delta y / 2}{1+c_{y}^{+} \Delta y / 2} \cdot \frac{1}{(\Delta y)^{2}}$ \\
\hline & \multirow[t]{4}{*}{$z_{k-1}$} & interior & $\left(\frac{\sigma_{V}}{\sigma_{H}}\right)^{2} \frac{1}{(\Delta z)^{2}}$ & & & \\
\hline & & $\lambda=0$ & & & & $-\left(\frac{\sigma_{V}}{\sigma_{H}}\right)^{2} \frac{1}{(\Delta z)^{2}}$ \\
\hline & & $\frac{\partial \lambda}{\partial z}=0$ & & & & $\left(\frac{\sigma_{Y}}{\sigma_{H}}\right)^{2} \frac{1}{(\Delta z)^{2}}$ \\
\hline & & $c_{z}^{-} \lambda-\frac{\partial \lambda}{\partial z}=0$ & & & . & $\frac{1-c_{z}^{-} \Delta z / 2}{1+c_{z}^{-} \Delta z / 2}:\left(\frac{\sigma_{Y}}{\sigma_{H}}\right)^{2} \frac{1}{(\Delta z)^{2}}$ \\
\hline & \multirow[t]{4}{*}{$z_{k+1}$} & interior & & & - & \\
\hline & & $\lambda=0$ & & & & $-\left(\frac{\sigma_{V}}{\sigma_{H}}\right)^{2} \frac{1}{(\Delta z)^{2}}$ \\
\hline & & $\frac{\partial \lambda}{\partial z}=0$ & & & & $\left(\frac{\sigma_{V}}{\sigma_{H}}\right)^{2} \frac{1}{(\Delta z)^{2}}$ \\
\hline & & $c_{z}^{+} \lambda+\frac{\partial \lambda}{\partial z}=0$ & & & & $\frac{1-c_{z}^{ \pm} \Delta z / 2}{1+c_{z}^{ \pm} \Delta z / 2} \cdot\left(\frac{\sigma_{V}}{\sigma_{H}}\right)^{2} \frac{1}{(\Delta z)^{2}}$ \\
\hline
\end{tabular}

Table 3: MATHEW stencil. The values of the stencil for any cell $n$ are found by summing contributions from all applicable rows. 
For improved convergence, preconditioning is used to modify the matrix $\mathbf{A}$ to one that is very close to the identity matrix I except for a few extreme eigenvalues. This is done by multiplying $\mathbf{A}$ by an easily calculable sparse symmetric matrix $\tilde{\mathbf{A}}^{-1}$

$$
\begin{aligned}
\tilde{\mathbf{A}}^{-1} \mathbf{A} & \approx \mathbf{I} \\
\tilde{\mathbf{A}}^{-1} \mathbf{A} \vec{x} & =\tilde{\mathbf{A}}^{-1} \vec{b} .
\end{aligned}
$$

As a first approximation, diagonal scaling

$$
\tilde{\mathbf{A}}_{i, j}=\frac{1}{\mathbf{A}_{i, j}} \delta_{i, j}
$$

is used for preconditioning.

The numerical algorithm [6] uses an initial guess for $\vec{x}_{1}$, which is an arbitrary approximation to the solution vector and may be taken to be the zero vector. The first residual $\vec{r}_{1}$, the preconditioned residual $\vec{z}_{1}$, and an initial direction vector $\vec{p}_{1}$ are generated according to

$$
\begin{aligned}
& \vec{r}_{1}=\left(\vec{b}-\mathbf{A} \vec{x}_{1}\right) \\
& \vec{z}_{1}=\tilde{\mathbf{A}}^{-1} \vec{r}_{1} \\
& \vec{p}_{1}=\vec{z}_{1} .
\end{aligned}
$$

The minimization then proceeds for successive $k$ by finding the $\alpha_{k}$ that minimize $f\left(\vec{x}_{k}+\right.$ $\alpha_{k} \vec{p}_{k}$ ) and generating the next search direction vector by $\mathbf{A}$-orthogonalization of the residuals according to the prescription

$$
\begin{aligned}
\alpha_{k} & =\frac{\vec{r}_{k} \cdot \vec{z}_{k}}{\vec{p}_{k} \cdot \mathbf{A} \cdot \vec{p}_{k}} \\
\vec{r}_{k+1} & =\vec{r}_{k}-\alpha_{k} \mathbf{A} \cdot \vec{p}_{k} \\
\vec{z}_{k+1} & =\tilde{\mathbf{A}}^{-1} \cdot \vec{r}_{k+1} \\
\vec{x}_{k+1} & =\vec{x}_{k}-\alpha_{k} \vec{p}_{k} \\
\beta_{k} & =\frac{\vec{r}_{k} \cdot \vec{z}_{k+1}}{\vec{r}_{k} \cdot \vec{z}_{k}} \\
\vec{p}_{k+1} & =\vec{z}_{k}-\beta_{k} \vec{p}_{k} .
\end{aligned}
$$

The residuals and direction vectors are mutually orthogonal and conjugate

$$
\begin{array}{rll}
\vec{r}_{i} \cdot \vec{r}_{j} & =0 & j<i \\
\vec{r}_{i} \cdot \vec{p}_{j} & =0 & j \leq i \\
\vec{p}_{i} \cdot \mathbf{A} \cdot \vec{p}_{j} & =0 & j<i
\end{array}
$$


so that each iteration proceeds by making the residual orthogonal to one more independent conjugate direction vector. The residuals $\vec{r}_{k}$ are also linearly independent and give the direction of steepest descent of $f(\vec{x})$ at each step. It should be noted that the conjugate direction vectors $\vec{p}_{k}$ are not given beforehand. Instead, they are constructed as linear combinations of the $\vec{r}_{k}$ by determination of the appropriate $\beta_{k}$ at each iteration.

Any of several convergence criterion can be adopted depending on the application. We have chosen a combination of

$$
\begin{aligned}
\left|\mathrm{A} \dot{\vec{x}}_{k}-\vec{b}\right| & <\Delta_{\text {tolerance }} \\
\frac{\left|\vec{x}_{k+1}-\vec{x}_{k}\right|}{\left|\vec{x}_{k+1}\right|} & <\Delta_{\text {tolerance }}
\end{aligned}
$$

The first tolerance check is used to stop the iterations, whenever the second condition is satisfied. These criteria are not completely robust; the final field should only be trusted when the solution convergences monotonically.

\section{Applications}

The optimized conjugate gradient code is more than twice as fast as the original assembly language successive overrelaxation algorithm. Using ADJUST_NORMAL and terrain FIXED_NORMAL boundary conditions, the new MATHEW yields the same results as the old, to the level of accuracy specified by the tolerance parameter, for assorted cell aspect and

$\frac{\sigma_{V}}{\sigma_{H}}$ ratios. However, the greater flexibility provided by MIXED boundary conditions leads to significant improvements in matching potential flow solutions in both two and three dimensions. The new boundary conditions can also be used to prevent artificial reductions in speed, to generate different flow patterns, and to eliminate unphysical wind field structures in cases of real complex terrain. We discuss several examples which illustrate these points.

\subsection{Two-dimensional flow around cylinder}

We first show results for two-dimensional flow around a cylindrical obstacle. Uniform winds in the $\mathrm{x}-\mathrm{y}$ plane are taken as the initial field with the ratio $\frac{\sigma_{Y}}{\sigma_{H}}$ set to $10^{-6}$ in order to effectively suppress vertical adjustments. Since both the velocity far from the cylinder and the MATHEW adjustment are irrotational, the resulting field should be the potential fiow 

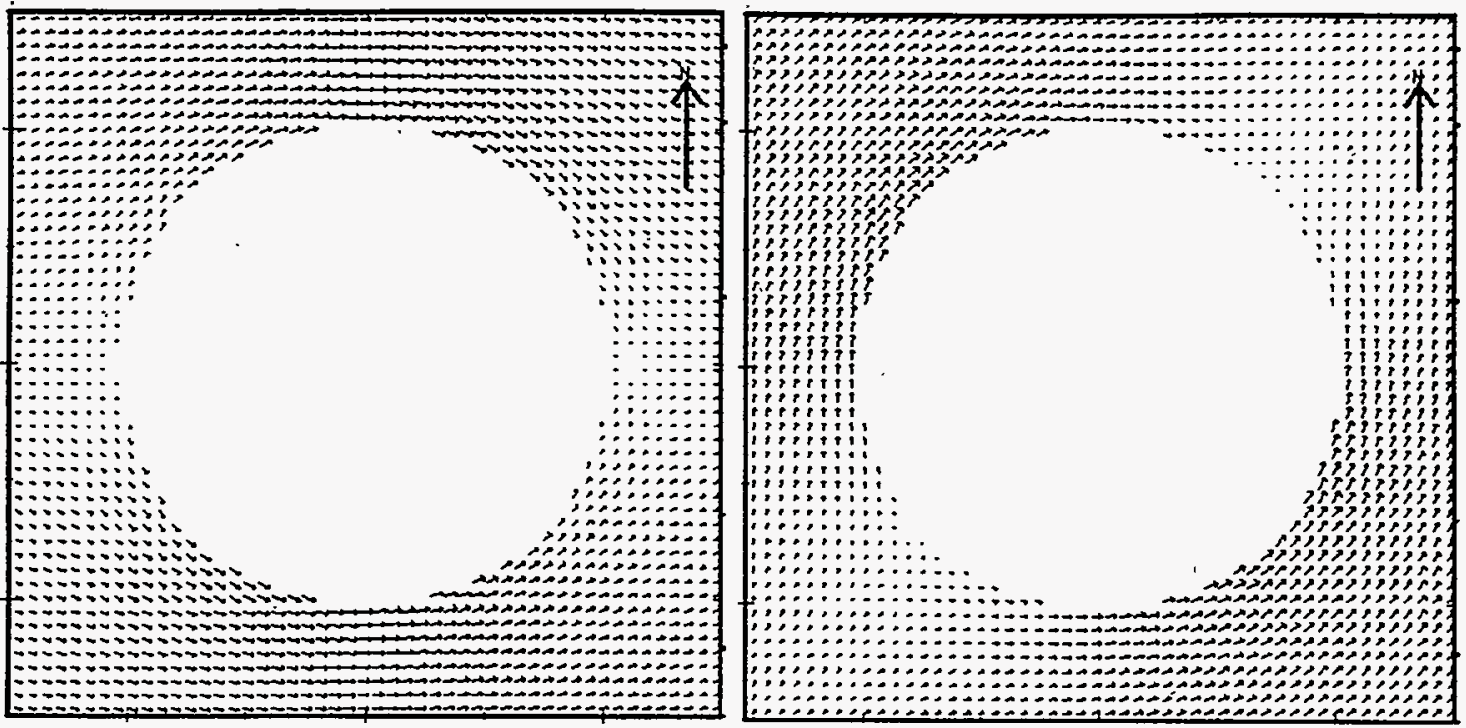

Figure 3: Two-dimensional potential flow solutions for a cylindrical obstacle. The left hand panel shows the flow solution for uniform winds impinging from the left, while the right hand panel shows the solution for winds at $225^{\circ}$.

solution for an irrotational incompressible fluid. For a cylinder of radius one and $\vec{u}^{\circ}=\hat{i}$, this is given by

$$
\begin{aligned}
\phi & =x+\frac{x}{r^{2}} \quad r \geq 1 \\
\vec{u} & =\nabla \phi=\left(1+\frac{1}{r^{4}}\left[y^{2}-x^{2}\right]\right) \hat{i}+\left(\frac{-2 x y}{r^{4}}\right) \hat{j} ;
\end{aligned}
$$

as can be verified by noting that $\phi$ satisfies

$$
u_{r}=\left.\frac{\partial \phi}{\partial r}\right|_{r=1}=\left.\cos \theta \frac{\partial}{\partial r}\left(r+\frac{1}{r}\right)\right|_{r=1}=0
$$

on the surface of the cylinder. The flow field for winds from any other direction can be found by a straightforward rotation. The potential flow solutions are shown for both $270^{\circ}$ and $225^{\circ}$ input winds in Figure 3.

To study the effect of lateral boundary conditions on the ability to reproduce potential flow, we performed simulations using ADJUST_NORMAL and MIXED boundary conditions with a range of values for the adjustment parameters $c_{x}^{+}=c_{x}^{-}=c_{x}$ and $c_{y}^{+}=c_{y}^{-}=c_{y}$. 

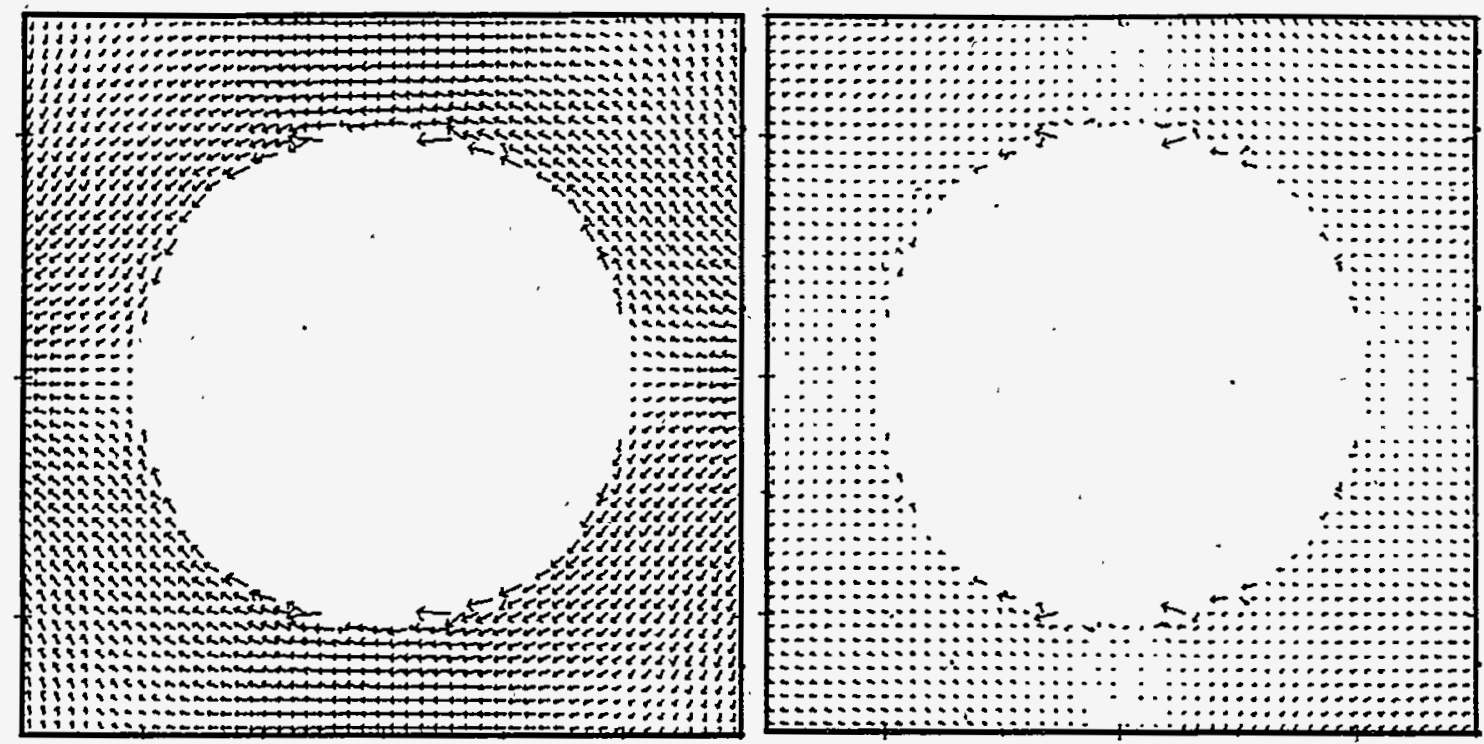

Figure 4: The difference between the potential flow and the MATHEW solution for $270^{\circ}$ winds and a cylindrical obstacle. The results for ADJUST_NORMAL and MIXED $\left(c=0.0008 \mathrm{~m}^{-1}\right)$ boundary conditions are shown on the left and right, respectively. The scale is exaggerated by a factor of five.

The simulation box is $100 \times 100$ cells with a uniform grid spacing of $30 \mathrm{~m}$, centered on a cylindrical obstacle 1000m in radius. The resulting vector field differences between the potential flow solution and the MATHEW results for $u_{0}=\hat{i}$ input winds are shown in Figure 4, where the magnitude of the difference vectors is exaggerated by a factor of five relative to Figure 3 for clarity. The ADJUST_NORMAL speeds are as much as $50 \%$ lower than the potential flow values, with the error increasing towards the lateral boundaries in certain directions. In comparison, the MIXED boundary solution, using an optimal parameter value of $c_{x}=c_{y}=c=0.0008 \mathrm{~m}^{-1}$, is a significantly improved match to potential flow except right at the surface of the cylinder, where some large discrepancies appear due to the use of a block terrain representation. The maximum speed difference is less than $5 \%$, dropping to $1 \%$ at the lateral boundaries. As $c \rightarrow \infty$ the solution approaches the ADJUST_NORMAL result, while in the opposite limit, the solution exhibits increásed speeds until the problem becomes overly constrained and fails to converge.

Figure 5 shows the difference vector fields for the two kinds of flow-through boundaries 

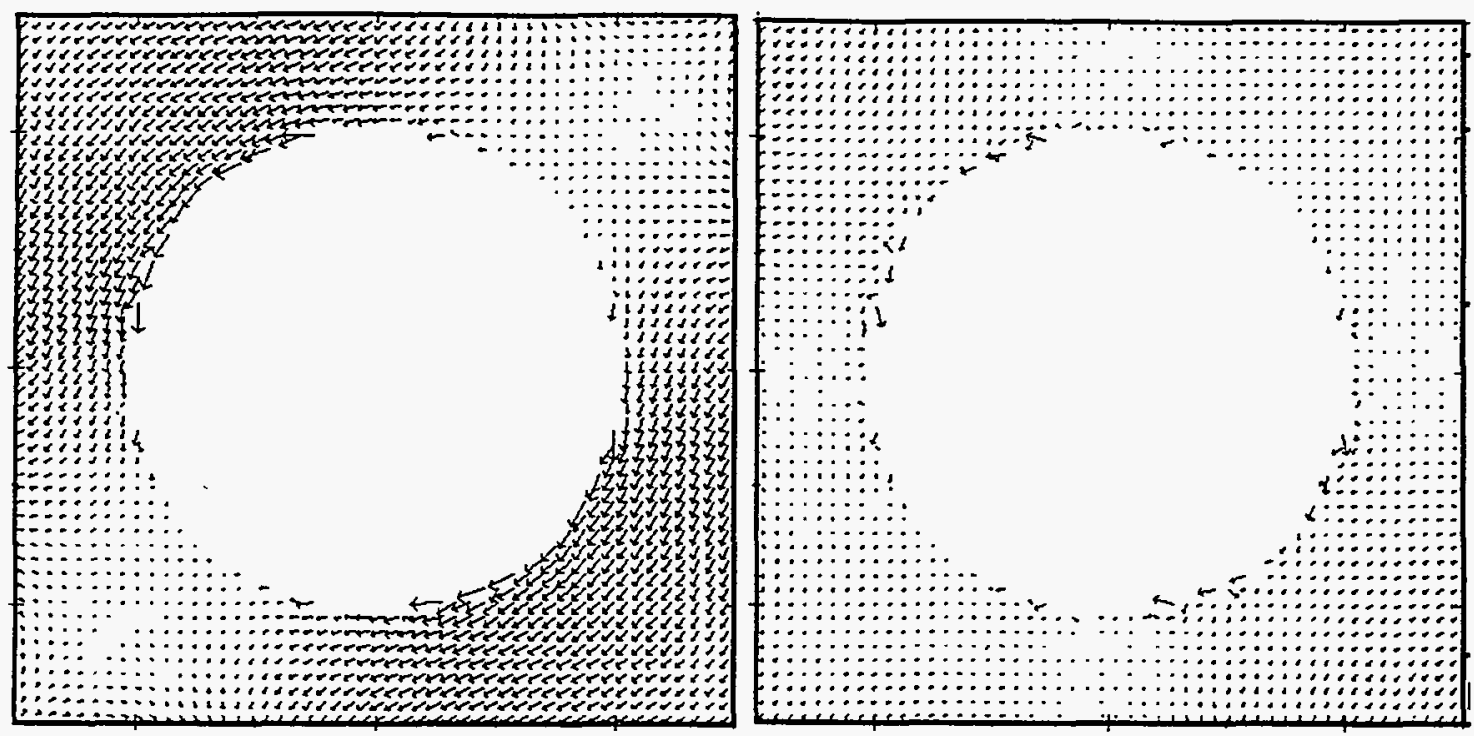

Figure 5: The difference between the potential flow and the MATHEW solution for $225^{\circ}$ winds and a cylindrical obstacle. The results for ADJUST_NORMAL and MIXED $\left(c=0.0008 \mathrm{~m}^{-1}\right)$ boundary conditions are shown on the left and right, respectively. The scale is exaggerated by a factor of five.

in the case of $225^{\circ}$ input winds. The ADJUST_NORMAL solution again exhibits reductions in speed on the order of 30-40\%. However, a comparison of the left hand panels of Figures 4 and 5 shows substantial differences. The ADJUST_NORMAL MATHEW result for $225^{\circ}$ winds matches the potential flow solution better for directions parallel to the input flow, but worse for normal directions. In contrast, the MIXED vector difference fields are comparable for the two input wind directions as expected by symmetry, apart from the $45^{\circ}$ rotation. The optimal MIXED boundary condition parameter $c$ is $0.0008 \mathrm{~m}^{-1}$ for both cases, resulting in speed reductions of $<5 \%$.

ADJUST_NORMAL boundary conditions exhibit considerable sensitivity to the size and orientation of the simulation box, while the MIXED condition is considerably more robust. Of course, for both conditions, the smaller the obstacle is relative to the simulation box, the smaller the boundary effects. Dimensionally, the MIXED-boundary parameter $c$ required to match the potential flow solution is an inverse length. Changing the grid resolution or the number of cells in one or more axis directions, the direction of the input winds fields, 

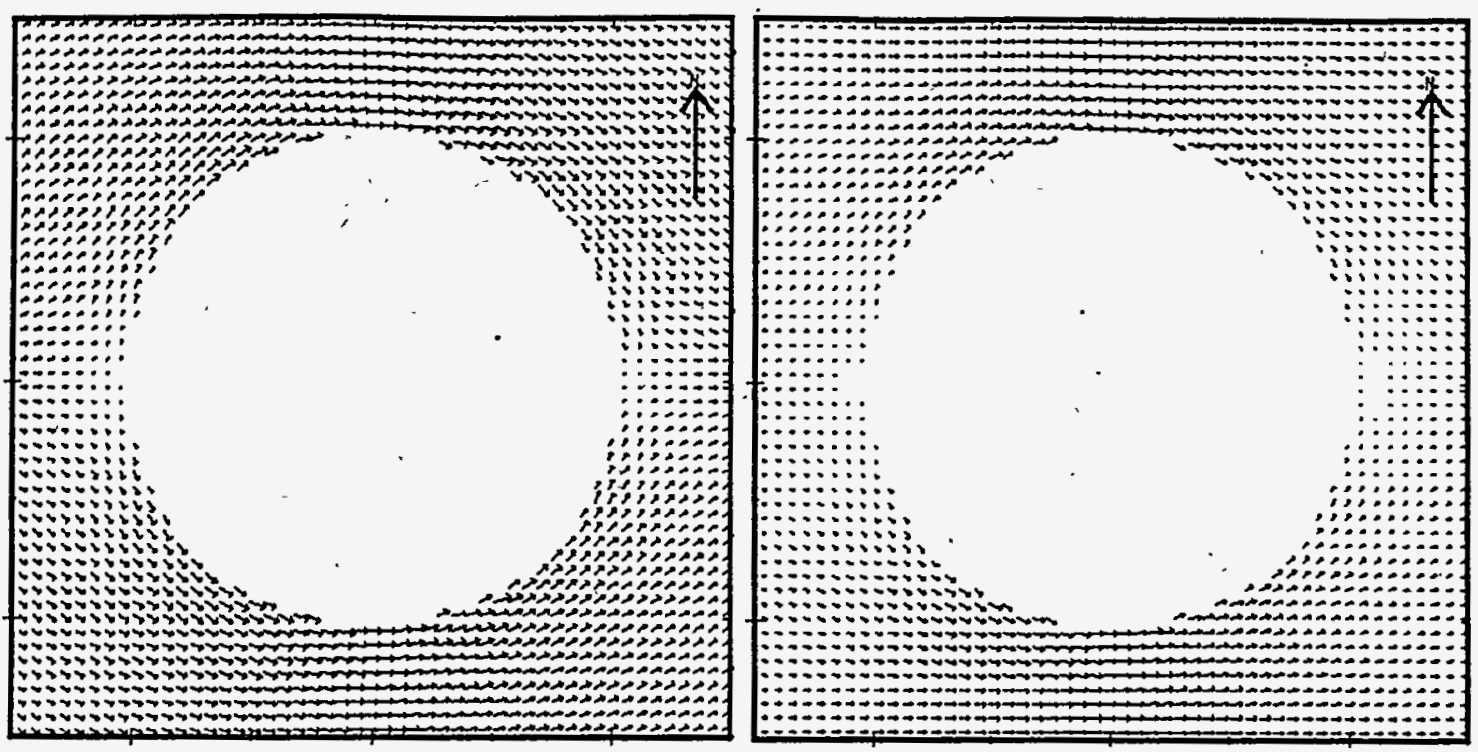

Figure 6: Wind fields solution for FIXED_NORMAL boundary conditions applied on the left and right border's with MIXED boundary conditions on the top and bottom (left hand) and vice versa (right).

or the size of the cylinder relative to the simulation box, does not lead to any significant difference in the magnitude of $c$. The only scale of relevance must therefore be that of the obstacle. The best matches to the potential flow solution use a MIXED parameter value of $0.0008 \mathrm{~m}^{-1}$, which is very close to the inverse cylindrical radius $(1000 \mathrm{~m})^{-1}$.

Neither ADJUST_NORMAL or FIXED_NORMAL boundary condition can replicate potential flow solutions since they restrict the degrees of freedom available for adjustment, distorting the winds in ways depending on both the geometry and the nature of the input field. Figure 6 demonstrates the effects of fixing the normal component of the wind field on different pairs of boundaries for the cylindrical obstacle with $270^{\circ}$ input winds. If MIXED conditions are applied on the top and bottom and FIXEB_NORMAL conditions on on the left and right hand sides of the box, the normal $(u)$ component of the wind on the latter pair of boundaries is maintained at the input value of $1 \mathrm{~m} / \mathrm{s}$. Adjustment in the tangential component then leads to increased wind speeds. In contrast, if FIXED_NORMAL conditions are instead applied to the top and bottom sides, the original zero normal component is maintained. Physically, this represents flow past a cylinder trapped in a horizontal channel. 

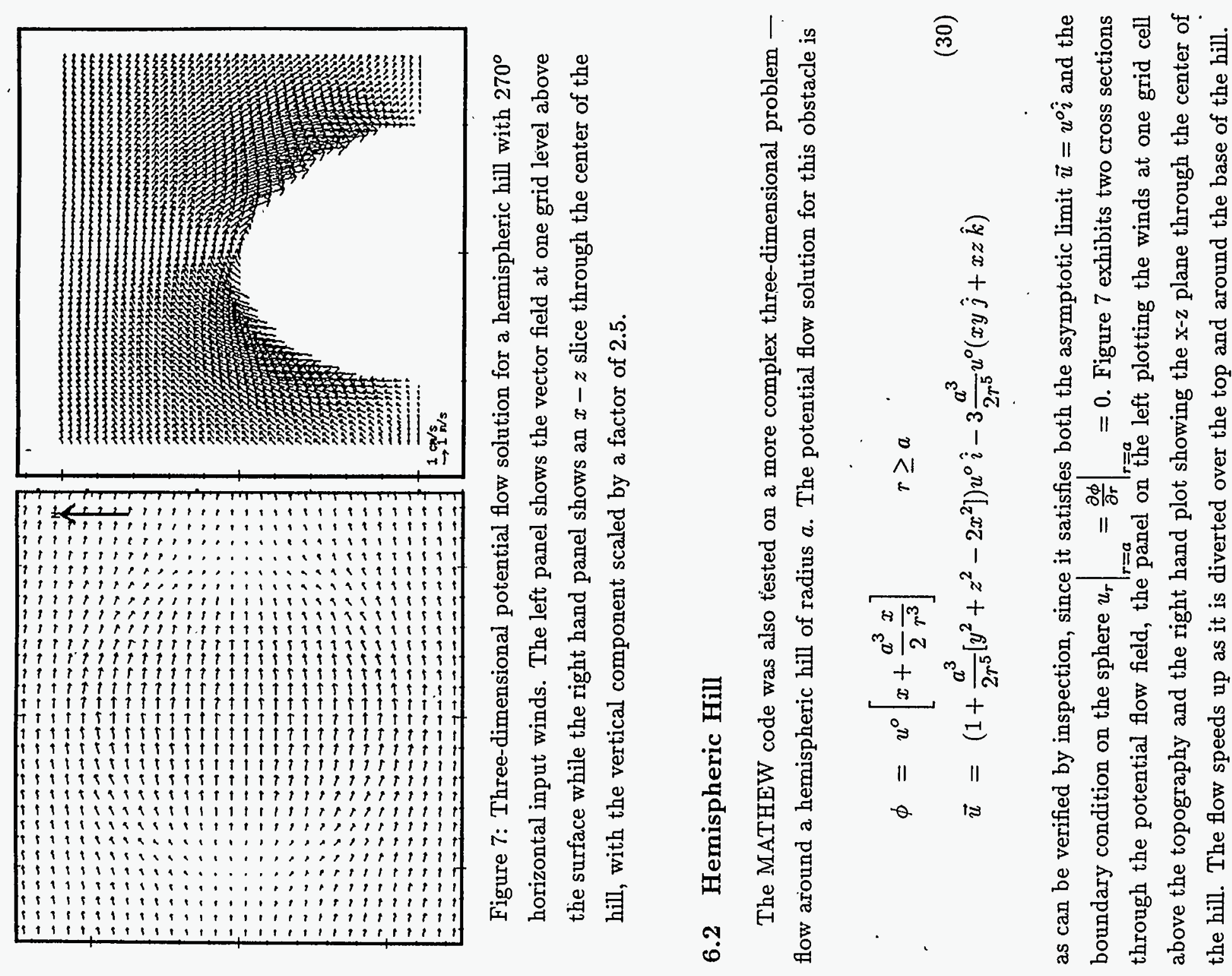

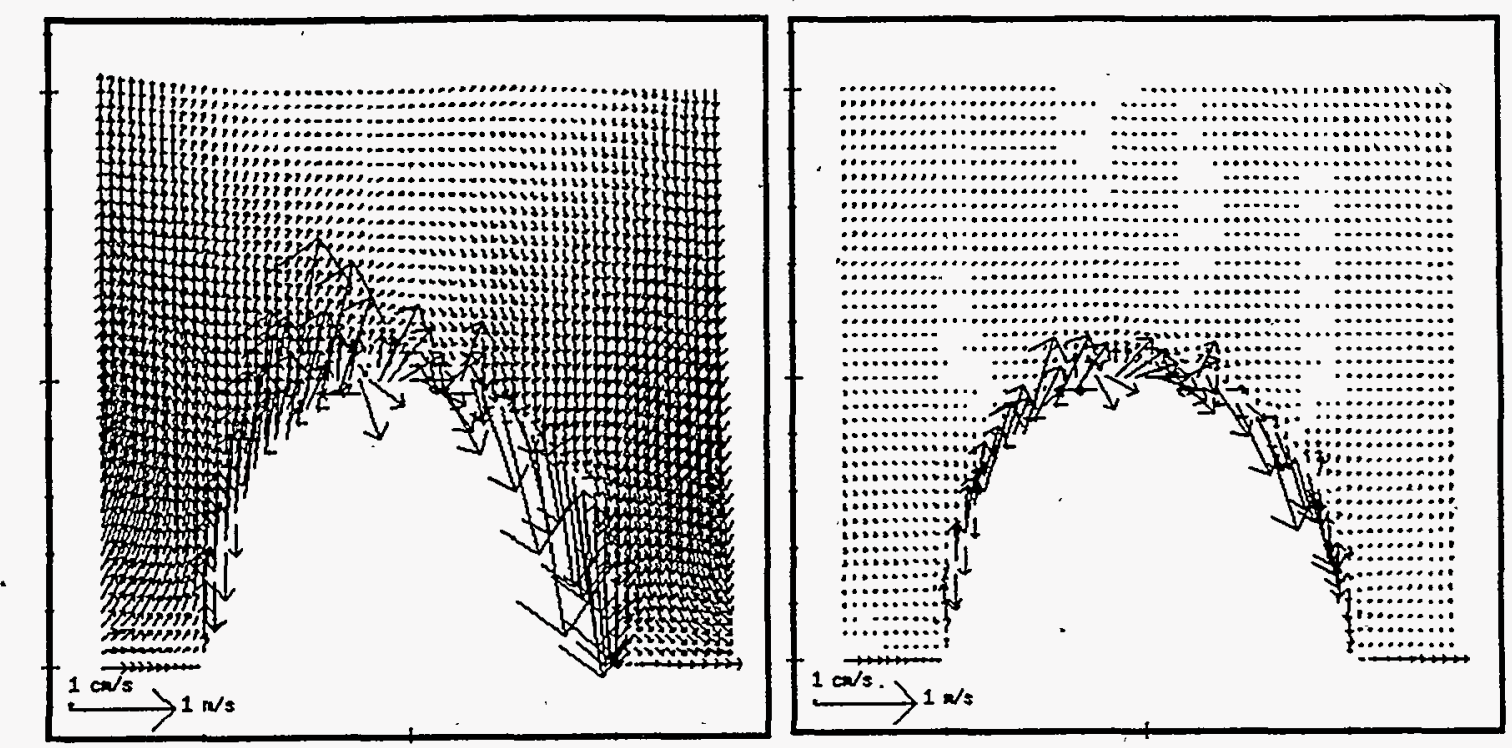

Figure 8: The difference between the potential flow solution and the MATHEW solution for ADJUST_NORMAL (left) and MIXED $c=0.003 \mathrm{~m}^{-1}$ (right) boundary conditions. An $x-z$ slice through the center of the hemispheric hill is shown, with the vertical component scaled by 2.5 and the vector lengths exaggerated by a factor of 5.

The simulation box is $60 \times 60 \times 40$ cells with a uniform spacing of $25 \mathrm{~m}$ in all dimensions, centéred on a hemispheric hill $500 \mathrm{~m}$ in radius. The ratio $\frac{\sigma_{Y}}{\sigma_{H}}$ is set to one to make adjustment equally likely in the vertical and the horizontal. As in the two-dimensional case, we are concerned with the ability of the algorithm to reproduce potential flow using a variety of both ADJUST_NORMAL and MIXED boundary conditions.

The difference between the MATHEW and the potential flow solutions is shown most clearly in the $x-z$ wind slice through the center of the hill. Figure 8 illustrates the vector difference fields for ADJUST_NORMAL and MIXED boundary conditions. Speeds are reduced up to $30 \%$ in the ADJUST_NORMAL case, even ignoring the large differences adjacent to the surface of the hill which are due to the block terrain representation. In contrast, the difference in speed is at most a few percent in directions along the flow, increasing to $10 \%$ at right angles for the MIXED boundary condition, using the optimal MIXED parameter $c=0.003 \mathrm{~m}^{-1}$. Input winds at $225^{\circ}$ yield similar results, the ADJUST_NORMAL boundary 

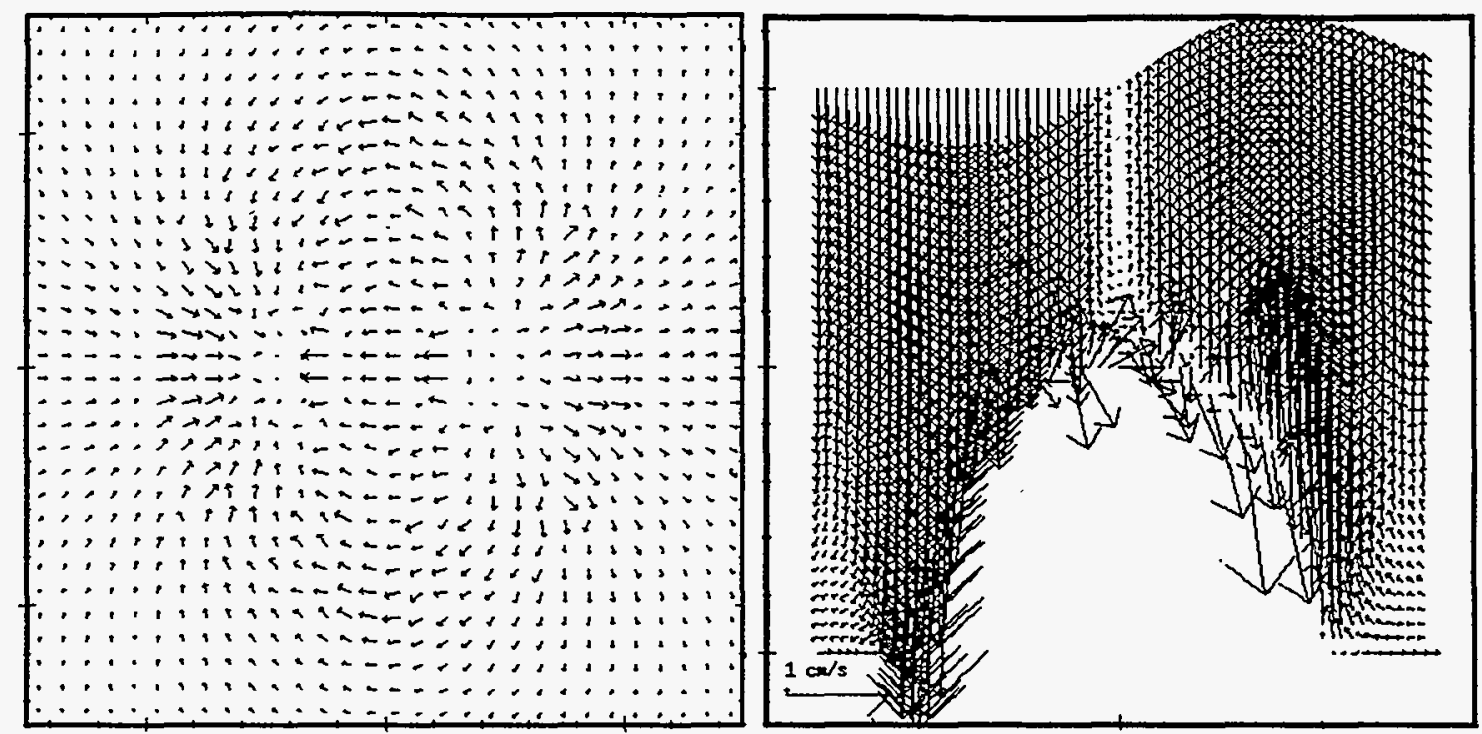

Figure 9: The difference between the potential flow solution and the MATHEW solution for a $\frac{\sigma_{V}}{\sigma_{H}}$ ratio of two. The left panel shows the field at one grid level above the surface while the right hand panel shows the $x-z$ slice.

performing significantly worse than the MIXED condition with greater speed reductions across the entire domain. Changes in the grid parameters or the size of the obstacle relative to the simulation box have no significant effect on the optimal value of $c, 0.002-0.003 \mathrm{~m}^{-1}$, which is in good agreement with the inverse length scale of the hemispherical hill $1 / 500 \mathrm{~m}^{-1}$.

A $\frac{\sigma_{Y}}{\sigma_{H}}$ ratio of two has been taken to be the condition of neutral atmospheric stability in MATHEW. However, the derivation of the variational algorithm and the simulation results indicate that, apart from a correction for the cell aspect ratio as determined by the $\Delta x, \Delta y$ and $\Delta z$ dimensions of the grid cell, the potential flow solution requires $\frac{\sigma_{V}}{\sigma_{H}}=1$. Figure 9 shows the difference vector fields for the hemispheric hill problem at one cell above topography and in the central X-z slice using MIXED boundary conditions and a $\frac{\sigma_{V}}{\sigma_{H}}$ ratio of two. Comparison with Figure 8 reveals that a much greater degree of vertical adjustment occurs, resulting in a large discrepancy with the potential flow solution. MATHEW converges $15-20 \%$ faster for a $\frac{\sigma_{V}}{\sigma_{H}}$ ratio of one then for a ratio of two, the more rapid convergence reflecting a choice of parameters allowing a greater degree of freedom in the adjustment. 

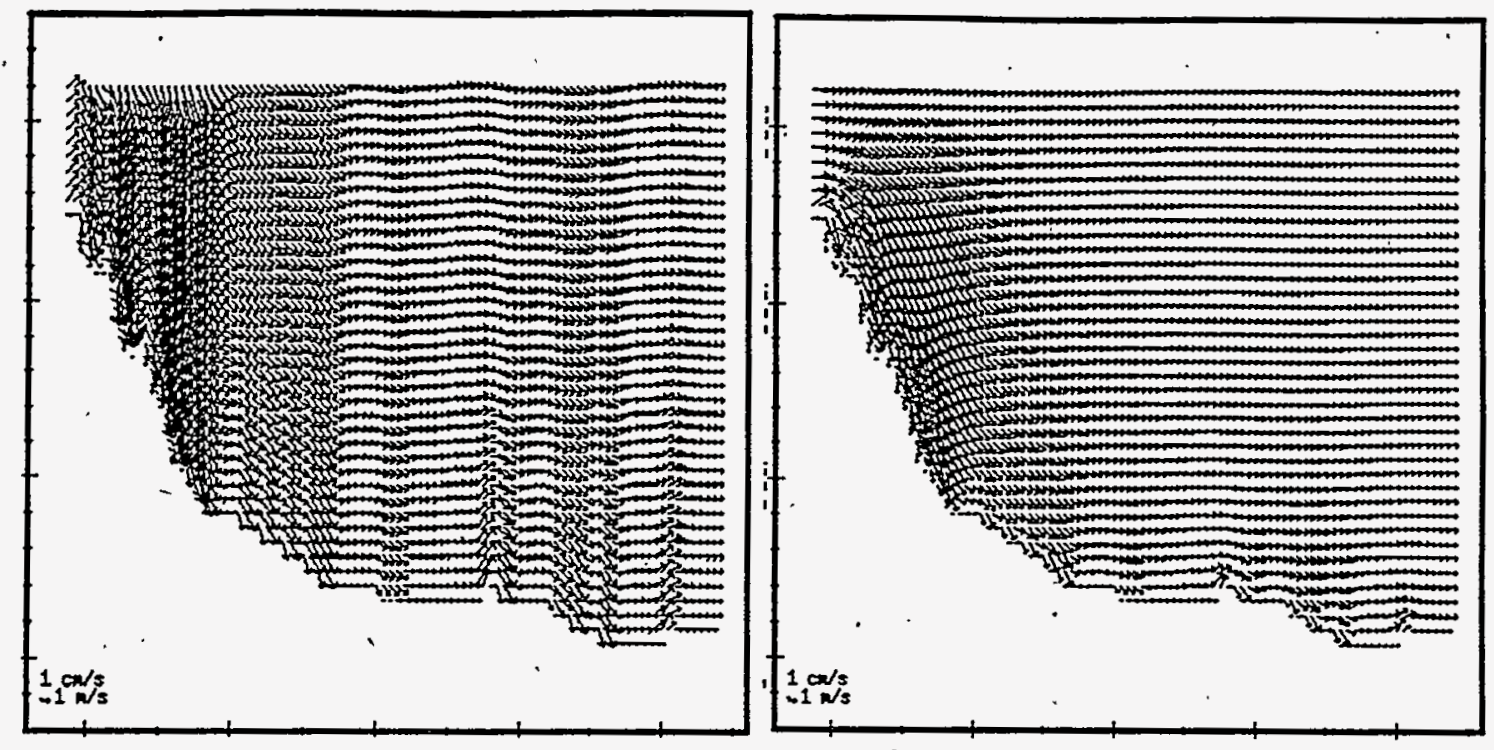

Figure 10: Rocky Flats simulation for uniform $3 \mathrm{~m} / \mathrm{s}$ winds at $270^{\circ}$. The left panel shows the results for ADJUST_NORMAL boundary conditions on both the top and lateral sides, while the right panel has a MIXED boundary parameter $c_{z}=0.0001 \mathrm{~m}^{-1}$ on the top. The vertical velocities are scaled by a factor of ten.

\subsection{Rocky Flats}

The 1991 Atmospheric Studies in Complex Terrain (ASCOT) field experiment was staged in the vicinity of the Rocky Mountain front range in Colorado. We use a simulation box appropriate to this problem, which.is bounded on the west by the crest of the Rockies, stretching out into the Great Plains to the east. The box is $80 \times 80 \times 40$ cells with a horizontal resolution of $0.5 \mathrm{~km}$ and a vertical resolution of $40 \mathrm{~m}$. Both uniform $3 \mathrm{~m} / \mathrm{s}$ input winds at $270^{\circ}$ and wind fields generated by interpolation and extrapolation from observed wind values are used. The $\frac{\sigma_{Y}}{\sigma_{H}}$ ratio is a value empirically determined to be appropriate for a nocturnal flow.

Figure 10 exhibits the effects of the complex terrain on the adjustment procedure for uniform $270^{\circ}$ input winds. A common problem of the ADJUST_NORMAL boundary conditions is the generation of unphysical inflows and outflows through the top of the grid, resulting in oscillatory patterns in the wind fields. While such structures may actually exist in the lee of the Rockies, they are not present in the input wind fields and variational relaxation 
cannot generate them on any physical or mathematical basis. Use of the MIXED parameter demonstrates that the waves are an artifact of the top boundary condition since they can be completely eliminated using a value of $c_{z}=0.0001 \mathrm{~m}^{-1}$. This requires twice as much convergence time as the ADJUST_NORMAL case. Applying MATHEW with ADJUST_NORMAL boundaries to the observationally generated input wind fields also leads to oscillatory patterns, which can be virtually removed using the MIXED condition with the same value of $c_{z}$. However, this requires 3-4 times the number of iterations as the ADJUST_NORMAL case, due to the existence of considerably larger inflows and outflows and additional adjustments required by the complex features of the wind field. Closing the top, by letting $c_{z} \rightarrow 0$, prevents any net vertical flow, but requires an order of magnitude more iterations.

The Rocky Mountains present a barrier to the flow varying between $1000-1600 \mathrm{~m}$ in height and $10 \mathrm{~km}$ in width. This would imply MIXED parameter values $c_{z}$ in the range $0.0001-0.001 \mathrm{~m}^{-1}$. However, in such a complex flow and terrain problem, it is not possible to ascertain a best value for the adjustment constant.

\section{Conclusion}

The conjugate gradient algorithm provides a robust means of solving the variational relaxation problem for wind fields. A straightforward implementation is at least twice as fast as an assembly language successive overrelaxation scheme running on a VAX platform, with additional speed up possible on vectorized or parallelized systems. The algorithm has the ability to control the relative amount of vertical and horizontal adjustment in order to reflect atmospheric stability conditions and incorporates a cell aspect correction to properly treat the fluxes through cell faces of varying areas. It can be applied to two- or three-dimensional input velocity fields supplied by diagnostic or prognostic models.

The MIXED boundary condition is derived from an additional surface term in the variational functional. It provides a means of controlling the relative amounts of tangential and normal adjustment of the winds on the lateral and top faces. Appropriate choices of the MIXED parameter $c$ can be used to generate different kinds of output winds fields, duplicate potential flow solutions, and eliminate unphysical structures introduced by complex terrain. The optimal value of $c$ is on the order of the inverse length scale of the obstacle for analytic geometries. However, in complex terrain problems, a priori determination of $c$ is difficult. It is possible to overly constrain the problem by using a MIXED parameter which is too 
restrictive and does not allow inflow and outflow to be balanced. However, the value of $c$ for which this occurs depends on characteristics of the input wind field as well as the nature of the topography.

Blocks provide a poor representation of terrain, causing unphysical wind fields due to flow blockage and distortion. The biggest discrepancies occur in the slip velocities, with the errors diminishing rapidly away from the surface. The conjugate gradient algorithm has been formulated to allow input winds with non-zero normal components at terrain boundaries, the surface constraint forcing the appropriate adjustment of the velocities. It

- is straightforwardly adaptable to continuous terrain representations.

\section{Acknowledgements}

We would like to thank D. Rodriguez for helpful discussions and for reviewing this paper. 


\section{References}

[1] Y. Sasaki, Mon. Wea. Rev 98, 875-898 (1970)

[2] C. Sherman, J. App. Met 17, 312-319 (1978)

[3] P. Gresho, UASG 75-22 (1975)

[4] R. Courant and D. Hilbert, Methods of Mathematical Physics, Interscience Publishers, John Wiley and Sons, New York (1962)

[5] R. Varga, Matrix Iterative Analysis, Prentice Hall, Englewood Cliffs, New Jersey (1962)

[6] W. Press, S. Teukolsky, W. Vetterling, and B. Flannery, Numerical Recipes, 2nd. ed., Cambridge University Press, Cambridge, U.K. (1993) 


\section{Appendix : MANUAL PAGES}

\subsection{MATHEW_CONTROL NAMELIST}

print_sides, Flag ('y' or 'n') which controls the printing of the three-dimensional arrays identifying the cell side classifications.

print_iccg_check. Flag ('y' or ' $n$ ') which controls the printing of the divergence checks on the conjugate-gradient solution.

The variable print_descriptors is no longer used.

\subsection{MATHEW_PARAMS NAMELIST}

conv_tolerance. Floating point value specifying the required convergence tolerance limit for the conjugate-gradient solver.

(Default: conv_tolerance $=0.01$ )

elliptic_eqn_test. Flag (' $y$ ' or ' $n$ ') which controls whether the code sets up and runs an elliptic equation test based on sinusoidal fields to test the implementation of boundary conditions and the conjugate-gradient solver

(Default: elliptic_eqn_test $=$ ' $n$ ')

fix_latieral_inflow. Flag ('y' or ' $n$ ') which controls whether the inflow is fixed on all lateral . faces.

(Default: fix_lateral_inflow $={ }^{\prime} n$ ')

fix_xmax_inflow. Flag ('y' or ' $n$ ') which controls whether the inflow is fixed on the maximum index $x$ face. Overridden if fix_lateral_inflow is set.

(Default: fix_xmax_inflow $=$ ' $n$ ')

fix_xmin_inflow. Flag. ('y' or ' $n$ ') which controls whether the inflow is fixed on the minimum index $x$ face. Overridden if fix_lateral_inflow is set.

(Default: fix_xmin_inflow $=$ ' $r$ ')

fix_ymax inflow. Flag ('y' or ' $n$ ') which controls whether the inflow is fixed on the maximum index $y$ face. Overridden if fix_lateral_inflow is set.

(Default: fix_ymax_inflow $=$ ' $n$ ') 
fix.ymin_inflow. Flag (' $y$ ' or 'n') which controls whether the inflow is fixed on the minimum index y face. Overridden if fix_lateral_inflow is set.

(Default: fix_ymin_inflow $=$ 'n')

iter_halt. Integer value specifying the maximum allowed number of iterations for the conjugate gradient solver.

(Default: iter_halt $=1500)$

lateral_boundary. 15-character string specifying the boundary condition on all lateral faces. The choices of boundary condition are 'ADJUST_NORMAL ', 'FIXED_NORMAL ', and 'MIXED'.

(Default: lateral_boundary = 'ADJUST_NORMAL')

mixed_lateral_adjust. Floating point value in inverse meters specifying the relative amount of normal to tangential adjustment for MIXED boundary conditions on all lateral faces. (Default: mixed_lateral_adjust $=1.0$ )

mixed_top_adjust. Floating point value in inverse meters specifying the relative amount of normal to tangential adjustment for MIXED boundary conditions on the top face. (Default: mixed_top_adjust $=1.0$ )

mixed_xmax_adjust. Floating point value in inverse meters specifying the relative amount of normal to tangential adjustment for MIXED boundary conditions on the maximum index $\mathrm{x}$ face. Overridden if mixed_lateral_adjust is set.

(Default: mixed_xmax_adjust $=1.0$ )

mixed_xmin_adjust. Floating point value in inverse meters specifying the relative amount of normal to tangential adjustment for MIXED boundary conditions on the minimum index $\mathrm{x}$ face. Overridden if mixed_lateral_adjust is set.

(Default: mixed_xmin_adjust $=1.0$ )

mixed_ymax_adjust. Floating point value in inverse meters specifying the relative amount of normal to tangential adjustment for MIXED boundary conditions on the maximum index $y$ face. Overridden if mixed_lateral_adjust is set.

(Default: mixed_ymax_adjust $=1.0$ ) 
mixed_ymin_adjust. Floating point value in inverse meters specifying the relative amount of normal to tangential adjustment for MixED boundary conditions on the minimum index y face. Overridden if mixed_lateral_adjust is set.

(Default: mixed_ymin_adjust $=1.0$ ) .

top_boundary. 15-character string specifying the boundary condition on the top boundary. The choices of boundary condition are 'ADJUST_NORMAL ', 'FIXED_NORMAL ', and 'MIXED'.

(Default: top_boundary = 'ADJUST_NORMAL ')

xmax_bourdary. 15-character string specifying the boundary condition on the maximum index $x$ face. The choices of boundary condition are 'ADJUST_NORMAL ', 'FIXED_NORMAL ', and 'MIXED'. Overriden if lateral_boundary is set.

(Default: xmax_boundary = 'ADJUST_NORMAL')

xmin_boundary. 15-character string specifying the boundary condition on the minimum index $x$ face. The choices of boundary condition are 'ADJUST_NORMAL ', 'FIXED_NORMAL ', and 'MIXED'. Overriden if lateral_boundary is set.

- (Default: xmin_boundary = 'ADJUST_NORMAL ')

ymax_boundary. 15-character string specifying the boundary condition on the maximum index $y$ face. The choices of boundary condition are 'ADJUST_NORMAL ', 'FIXED_NORMAL ', and 'MIXED'. Overriden if lateral_boundary is set.

(Default: ymax_boundary' = 'ADJUST_NORMAL')

ymin_boundary. 15-character string specifying the boundary condition on the minimum index $y$ face. The choices of boundary condition are 'ADJUST_NORMAL ', 'FIXED_NORMAL ', and 'MIXED'. Overriden if lateral_boundary is set.

(Default: ymin_boundary = 'ADJUST_NORMAL')

The variables convergence_error, over_relax, iter_over_relax_shift, and close_gridtop are no longer used. Note that iter_halt now applies to the conjugate gradient solver. The variable stab_mathew sets the ratio $\frac{\sigma_{V}}{\sigma_{H}}$ as twice the cell aspect ratio multiplied by a factor dependent on the atmospheric stability conditions. This has not been altered, but should be rethought in light of the discussion of neutral stability. 